\title{
ARTICLE \\ Influences of an NRII2 polymorphism on heterogeneous antiplatelet reactivity responses to clopidogrel and clinical outcomes in acute ischemic stroke patients
}

\author{
Yi-bei Chen ${ }^{1}$, Zi-yi Zhou ${ }^{2}$, Guo-min Li $^{2}$, Can-xing Xiao ${ }^{1}$, Wei-bang Yu', Shi-long Zhong ${ }^{3}$, Ye-feng Cai ${ }^{2}$, Jing Jin ${ }^{1}$ and Min Huang ${ }^{1}$
}

Pregnane $X$ receptor (PXR) is a member of nuclear receptor subfamily 1 (NR1/2) that is a transcriptional regulator of several metabolic enzymes involved in clopidogrel metabolism. In this study we identified and evaluated the contributions of single nucleotide polymorphisms (SNPs) in NR112 and cytochrome P450 (CYP) 2 C19 alleles to clopidogrel resistance (CR) and long-term clinical outcomes in acute ischemic stroke (IS) patients. A total of 634 patients with acute IS were recruited, who received antiplatelet medication (clopidogrel or aspirin) every day and completed a 1-year follow-up. The selected SNPs were genotyped, and platelet function was measured. Modified Rankin Scale (mRS) scores and main adverse cardiovascular and cerebrovascular events (MACCE) were noted to assess the prognosis. We showed that SNPs NR1/2 rs 13059232 and CYP2C19 alleles ( $\left.2^{*} / 3^{*}\right)$ were related to CR. SNP NR1/2 (rs13059232) was identified as an independent risk factor for the long-term clinical outcomes in the clopidogrel cohorts $(P<0.001)$, but similar results were not observed in a matched aspirin cohort $(P>0.05)$. Our results suggest that NR1/2 variant (rs13059232) could serve as biomarker for clopidogrel therapy and individualized antiplatelet medications in the treatment of acute IS patients.

Keywords: acute ischemic stroke; anti-platelet medication; clopidogrel; aspirin; NR1/2; individualized medication

Acta Pharmacologica Sinica (2019) 40:762-768; https://doi.org/10.1038/s41401-018-0178-4

\section{INTRODUCTION}

As one of the first-line antiplatelet agents, clopidogrel is extensively used for the treatment and prevention of ischemic stroke (IS) [1, 2]. Unfortunately, the individual differences in responses to clopidogrel-based therapy are significant, which seriously affects the safety and efficacy of its application [3]. In recent years, the mechanism underlying clopidogrel resistance (CR) has been investigated in many ways, including demographic factors, genetic factors, and medication [4-6]. However, the mechanisms of $C R$ have not been clearly explained.

As a thienopyridine prodrug, the antiplatelet effect of clopidogrel is dependent on hepatic transformation, and many genes related to its hepatic transformation affect the pharmacokinetic response to clopidogrel [7-9]. Currently, research on interindividual variations in clopidogrel responses is mainly focused on the genetic polymorphisms of drug-metabolizing enzymes (CYP2C19, CYP3A4, CYP2C9, CYP2B6, and CES1) and transporters (ABCB1) [10-16]. However, the impact of known genetic variants could only explain a very limited portion of individual differences in $C R$ and the clinical outcomes of IS patients [17].

Pregnane $X$ receptor (PXR), encoded by the NR1/2 gene, belongs to the nuclear receptor superfamily, PXR universally regulates the activities of drug-metabolizing enzymes and drug transporters [18, 19]. NR1/2 genetic polymorphisms have a significant impact; specifically, they affect the uptake, transport, and metabolism of various endogenous and exogenous substances. Therefore, NR1/2 genetic polymorphisms are of great significance in studying the metabolic processing of clinical drugs [20, 21]. Nevertheless, research on the relationship between the genetic polymorphisms of NR1/2 and individual differences in the therapeutic effects of clopidogrel is currently limited.

In our previous studies, several single nucleotide polymorphism (SNPs) of metabolic enzymes and NR112 were found to be related to CR in acute IS patients; however, their influence on the longterm clinical outcomes of patients treated with clopidogrel and other antiplatelet drugs remains unclear [9]. The aim of the current study was to further investigate the impact of CYP2C19*2 rs4244285, CYP2C19*3 rs4986893, and NR112 rs13059232 on CR and long-term clinical outcomes in patients suffering from acute IS. This study will help determine the appropriate individualized antiplatelet medication.

\section{MATERIALS AND METHODS}

Study population

This study protocol, approved by the Ethics Committee of Guangdong Provincial Hospital of Traditional Chinese Medicine and Guangdong General Hospital, was compatible with the Declaration of Helsinki. Informed consents were obtained from

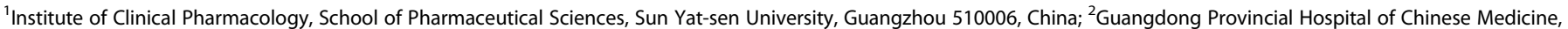
Guangzhou 510120, China and ${ }^{3}$ Medical Research Center of Guangdong General Hospital, Guangzhou 510080, China

Correspondence: Shi-long Zhong (zhongsl@hotmail.com) or Ye-feng Cai (caiyefeng@126.com) or Jing Jin (jinjing@mail.sysu.edu.cn)

Received: 13 June 2018 Accepted: 26 September 2018

Published online: 28 November 2018 
all individual participants included in the study. This study was a double-center observational trial.

Our research had three phases (Fig. 1). In phase 1, we investigated the association among relative factors, $C R$, and clinical outcomes. In this phase, 259 acute IS patients between 40 and 80 years of age were enrolled at the Guangdong Provincial Hospital of Traditional Chinese Medicine from March 2013 to October 2015. The inclusion criteria were as follows: (1) compliance with the diagnostic criteria of IS and verification by CT or MRI; (2) treatment with clopidogrel for at least 5 days during hospitalization; (3) informed consent signed by patients or their relatives; and (4) recruited within $24 \mathrm{~h}$ after stroke onset. The exclusion criteria were as follows: (1) silent cerebral infarction without obvious symptoms; (2) a platelet count $>450 \times 10^{9} / \mathrm{L}$ or $<150 \times 10^{9} / \mathrm{L}$; (3) long-term treatment with warfarin, heparin, ticlopidine, dipyridamole and proton pump inhibitors; and (4) functional lesion on the liver or kidney. Among these patients, 192 subjects (clopidogrel cohort 1) completed a 1-year follow-up and consistently took clopidogrel every day during 1 year. Platelet function was measured using the light transmission aggregometry method to evaluate the antiplatelet responses of these patients. Sequenom MassARRAY iPLEX technology was used to genotype the selected SNPs. Follow-up telephone interviews were conducted with patients at 6 months and 12 months after hospital discharge. In phase 2, 140 patients with acute IS at Guangdong General Hospital were enrolled to verify the results from the Guangdong Provincial Hospital of Traditional Chinese Medicine. Patients were recruited within $24 \mathrm{~h}$ after stroke onset and were all treated with a daily clopidogrel maintenance dose of $75 \mathrm{mg}$ during a 1-year follow-up (clopidogrel cohort 2). All of these patients completed follow-up telephone interviews and SNP genotyping. There was no significant difference in age, gender, diabetes, smoking, alcohol, or biochemical indexes among these two cohorts. In phase 3, an aspirin cohort of 302 IS patients who took a daily maintenance dose of $100 \mathrm{mg}$ of aspirin monotherapy during a 1-year follow-up (aspirin cohort) was enrolled from the two hospitals to study the influence of the selected SNPs on different antiplatelet agents. This aspirin cohort was matched with the clopidogrel cohort (including clopidogrel cohorts 1 and 2), including age, gender, diabetes, smoking, alcohol and biochemical indexes (Table 1). All 302 patients completed follow-up telephone interviews and SNP genotyping.

In total, 634 patients completed a 1-year follow-up and consistently took antiplatelet agents every day during 1 year, and their demographic characteristics, clinical medications, and biochemical indexes were collected from the electronic medical records of these two hospitals.

Platelet function testing and clopidogrel resistance

The light transmission aggregometry method was used to evaluate antiplatelet reactivity responses to clopidogrel. Peripheral blood samples were collected from patients before treatment and on days 5-7 after treatment. The maximal platelet aggregation rate (MPAR) was calculated by using an LBY-NJ4A automatic platelet aggregation analyzer with $10 \mu \mathrm{mol} / \mathrm{L}$ adenosine diphosphate (ADP) inducer. CR was defined as either less than a $10 \%$ change in the MPAR ( $d 5-7$ ) or an MPAR (d 5-7) $>50 \%$ of the baseline magnitude $(\mathrm{d} 0)$. The platelet inhibition rate (PIR) was quantified as follows: PIR $=$ PAR $\left(d_{0}\right)$-PAR $\left(d_{5}\right)$. PIR $<10 \% \times$ PAR $\left(d_{0}\right)$ or PAR $\left(d_{5}\right)>50 \%$ PAR $\left(d_{0}\right)$ was defined as CR.

Follow-up and clinical outcomes

Follow-up telephone interviews were conducted at 6 months and 12 months after hospital discharge, and the data were verified by

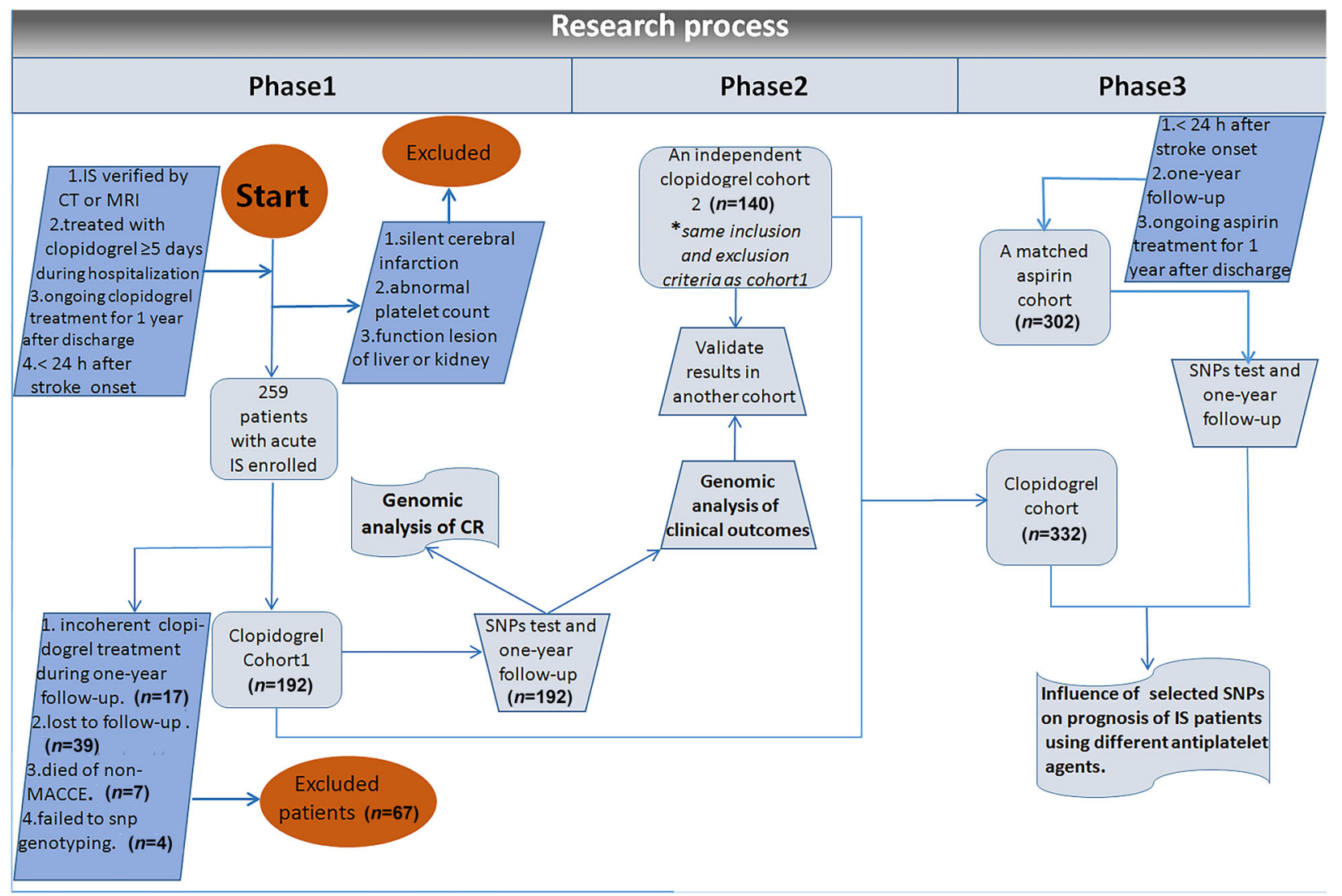

Fig. 1 Flow diagram of the experimental design 
Table 1. Characteristics and prognosis of the study population

\begin{tabular}{|c|c|c|c|c|c|c|c|c|c|}
\hline \multirow{2}{*}{$\begin{array}{l}\text { Clinical } \\
\text { parameters }\end{array}$} & \multicolumn{3}{|l|}{ Phase 1} & \multicolumn{3}{|l|}{ Phase 2} & \multicolumn{3}{|l|}{ Phase 3} \\
\hline & $\mathrm{NCR}$ & $\mathrm{CR}$ & $P$ value & $\begin{array}{l}\text { Clopidogrel } \\
\text { cohort } 1\end{array}$ & $\begin{array}{l}\text { Clopidogrel } \\
\text { cohort } 2\end{array}$ & $P$ value & $\begin{array}{l}\text { Clopidogrel cohort } \\
\text { (total) }\end{array}$ & $\begin{array}{l}\text { Aspirin } \\
\text { cohort }\end{array}$ & $P$ value \\
\hline Total cases, $n$ & $127(66.1 \%)$ & 65 (33.9\%) & - & 192 & 140 & - & 332 & 302 & - \\
\hline Age & $66.0 \pm 10.9$ & $67.0 \pm 13.1$ & 0.994 & $65.6 \pm 11.4$ & $65.7 \pm 12.4$ & 0.759 & $65.6 \pm 11.8$ & $63.6 \pm 11.3$ & 0.105 \\
\hline Sex Male, $n(\%)$ & 79 (62.2\%) & 38 (58.5\%) & 0.615 & 123 (64.1\%) & $92(65.7 \%)$ & 0.756 & $215(64.8 \%)$ & $202(66.9 \%)$ & 0.726 \\
\hline Female, $n(\%)$ & 48 (37.8\%) & 27 (41.5\%) & & 69 (35.9\%) & 48 (34.3\%) & & $117(35.2 \%)$ & $100(33.1 \%)$ & \\
\hline Diabetes, $n$ (\%) & 37 (29.1\%) & $20(30.8 \%)$ & 0.814 & 65 (33.9\%) & 51 (36.4\%) & 0.627 & 116 (34.9\%) & 98 (32.4\%) & 0.508 \\
\hline $\begin{array}{l}\text { Hypertension, } \\
\text { n (\%) }\end{array}$ & 75 (59.1\%) & 49 (75.4\%) & $0.025 *$ & 130 (67.7\%) & 95 (67.9\%) & 0.977 & $225(67.8 \%)$ & 211 (69.8\%) & 0.569 \\
\hline Smoke, $n(\%)$ & $36(28.3 \%)$ & $17(26.1 \%)$ & 0.748 & $50(26.0 \%)$ & 25 (17.9\%) & 0.078 & 75 (22.6\%) & 76 (25.2\%) & 0.447 \\
\hline Drink, $n(\%)$ & 12 (9.4\%) & 7 (10.8\%) & 0.772 & $18(9.4 \%)$ & 7 (5.0\%) & 0.136 & 25 (7.5\%) & 27 (8.9\%) & 0.518 \\
\hline TC (mmol/L) & $4.68 \pm 1.00$ & $4.74 \pm 1.27$ & 0.721 & $4.57 \pm 1.09$ & $4.86 \pm 1.44$ & 0.069 & $4.68 \pm 1.28$ & $4.70 \pm 1.22$ & 0.646 \\
\hline TG (mmol/L) & $1.61 \pm 0.86$ & $1.69 \pm 1.22$ & 0.506 & $1.65 \pm 1.16$ & $1.63 \pm 1.13$ & 0.904 & $1.64 \pm 1.15$ & $1.68 \pm 1.07$ & 0.650 \\
\hline LDL-C (mmol/L) & $1.15 \pm 0.31$ & $1.10 \pm 0.32$ & 0.183 & $2.89 \pm 0.97$ & $3.07 \pm 1.15$ & 0.170 & $2.97 \pm 1.06$ & $2.94 \pm 1.07$ & 0.640 \\
\hline $\mathrm{HDL}-\mathrm{C}(\mathrm{mmol} / \mathrm{L})$ & $3.01 \pm 0.89$ & $3.02 \pm 1.07$ & 0.889 & $1.12 \pm 0.33$ & $1.05 \pm 0.33$ & 0.077 & $1.09 \pm 0.34$ & $1.08 \pm 0.34$ & 0.467 \\
\hline $\begin{array}{l}\text { Vascular death } \\
\text { (1 year) }\end{array}$ & $9(7.1 \%)$ & $6(9.2 \%)$ & 0.600 & $15(7.8 \%)$ & $5(3.6 \%)$ & 0.109 & $20(6.0 \%)$ & $11(36.4 \%)$ & 0.165 \\
\hline MI/IS/TIA (1 year) & $17(13.4 \%)$ & $15(23.1 \%)$ & 0.088 & $32(16.7 \%)$ & $24(17.1 \%)$ & 0.909 & $56(16.9 \%)$ & 39 (12.9\%) & 0.164 \\
\hline mRS (1 year) & $1.70 \pm 1.83$ & $1.85 \pm 1.86$ & 0.600 & $1.75 \pm 1.84$ & $1.80 \pm 1.34$ & 0.799 & $1.78 \pm 1.65$ & $1.59 \pm 1.64$ & 0.157 \\
\hline $\begin{array}{l}\text { Poor outcome, } \\
\text { n (\%) (1 year) }\end{array}$ & 43 (33.9\%) & $32(49.2 \%)$ & $0.039 *$ & $73(38.0 \%)$ & 46 (32.9\%) & 0.333 & $119(35.8 \%)$ & 93 (30.8\%) & 0.303 \\
\hline
\end{tabular}

$N C R$ nonclopidogrel resistance, $C R$ clopidogrel resistance, $T I A$ transient ischemic attack, $T C$ Total cholesterol, $T G$ triglyceride, $L D L-C$ low density lipoprotein cholesterol, HDL-C high density lipoprotein cholesterol, MI myocardial infarction, TIA transient ischemic attack, mRS modified Rankin Scale ${ }^{*} P<0.05$

Bold values in tables are used to stress statistics with significant differences

information obtained from the case management system in the medical record room of the two hospitals. Poor outcomes were defined as a score of more than 2 on the modified Rankin Scale or the occurrence of a main adverse cardiovascular and cerebrovascular event (MACCE), including death, myocardial infarction, and additional strokes.

Genotyping analysis

DNA samples were obtained from the peripheral blood, and three SNPs in two genes were genotyped: NR112 (rs13059232), CYP2C19*2 (rs4244285), and CYP2C19*3 (rs4986893). DNA purity and concentration were determined on a NanoDrop 2000 (Thermo), and the absorbance ratios (A260/A280) of all DNA samples were between 1.6 and 1.9 to ensure quality. All of the selected SNPs were genotyped using Sequenom MassARRAY iPLEX technology (Sequenom, San Diego, CA, USA). DNA (10 ng) samples were amplified and extended using PCR amplification. A 384-pad SpectroCHIP and MALDI-TOF mass spectrometry (Sequenom) were used to detect alleles. MassArray Typer 4.0 software was used to collect and analyze the data.

Statistical analysis

Data are presented as the mean $\pm S D$ or in frequencies. Differences between cohorts were assessed by Pearson's chisquare test (when the variables were categorical) or one-way ANOVA (when the variables were continuous). Hardy-Weinberg equilibrium and allele frequency comparisons were performed by using the chi-square test. Determination of the mode of inheritance was analyzed by comparing genotype effects. The associations among $C R$, clinical outcomes, and related factors were analyzed using Pearson's chi-square test. CYP2C19*2/*3 $\left({ }^{*} 2 /{ }^{*} 3\right.$ carrier $=1$, noncarrier $=0)$ and NR1/2 rs13059232 (CC $=1, C T+T$ $=0$ ) were added to the logistic regression analysis of $C R$ and clinical outcomes to investigate the interaction between CYP2C19*2/*3 and NR1/2 rs13059232. The cumulative risk of MACCEs was assessed using a Kaplan-Meier survival curve. Sex (male $=1$, female $=0)$, age, hypertension (presence $=1$, absence $=0$ ), diabetes (presence $=1$, absence $=0)$, CYP2C19*2/*3 $\left({ }^{*} 2 /{ }^{*} 3\right.$ carrier $=1, \quad$ noncarrier $=0), \quad$ and $\quad$ NR112 rs13059232 $(C C=1, C T+\Pi=0)$ were added to the Cox regression analysis to investigate the risk factors for MACCEs. All statistical analyses were performed with SPSS version 21.0. Statistical significance was assumed for $P<0.05$.

\section{RESULTS}

Clinical characteristics of the study population

In phase 1, 259 acute IS patients from the Guangdong Provincial Hospital of Traditional Chinese Medicine were admitted to study the correlation among SNPs, CR, and clinical outcomes. A total of 192 subjects completed a 1-year follow-up and consistently took a daily clopidogrel maintenance dose of $75 \mathrm{mg}$ during 1 year (clopidogrel cohort 1). The excluded subjects are shown in Fig. 1. The CR rate was $33.9 \%(n=65)$ of the research population. Table 1 shows the clinical characteristics of the $C R$ group and the nonclopidogrel resistance (NCR) group. There were 117 males $(60.9 \%)$ and 75 females (39.1\%) included in our study. The average age was $66.5 \pm 11.7$ years. Among the 192 patients, $57(29.7 \%)$ had diabetes mellitus and 124 (64.6\%) had hypertension. There was no significant difference in age, gender, diabetes, smoking, or biochemical indexes among these cohorts, but the patients with hypertension had a higher risk of CR (75.4\% vs. $59.1 \%)$. According to the method described in "Follow-up and clinical outcomes", $38.0 \%(n=73)$ of these patients had poor outcomes. Patients who were identified as having $C R$ in the early period of IS had significantly poorer clinical outcomes during the 1-year follow-up 
Table 2. Correlation of clinical outcome with clopidogrel resistance

\begin{tabular}{llllll}
\hline & Poor outcome (\%) & Good outcome (\%) & Chi-square & $P$ value & OR \\
\hline CR & $32(49.2 \%)$ & $33(50.8 \%)$ & 4.268 & $\mathbf{0 . 0 3 9 *}$ & 1.894 \\
NCR & $43(33.9 \%)$ & $84(66.1 \%)$ & & & $(1.030-3.485)$ \\
\hline$N C R$ nonclopidogrel resistance, $C R$ clopidogrel resistance & & & \\
${ }^{*} P<0.05$ & & & & \\
\hline
\end{tabular}

Table 3. Correlation of selected SNPs with clopidogrel resistance

\begin{tabular}{|c|c|c|c|c|c|c|c|c|}
\hline Genes & SNPs & Genotypes & NCR (\%) & CR (\%) & Chi-square & $P$ value & OR & $95 \% \mathrm{Cl}$ \\
\hline \multirow[t]{2}{*}{$\mathrm{CYP} 2 \mathrm{C} 19 * 2 / * 3$} & rs4244285/ rs4986893 & Carriers & $65(60.2 \%)$ & $43(39.8 \%)$ & 4.764 & $0.029 *$ & 2.108 & $(1.069-3.808)$ \\
\hline & & Noncarrier & $61(75.3 \%)$ & $20(24.7 \%)$ & & & & \\
\hline & & $\mathrm{CC}$ & $37(55.2 \%)$ & $30(44.8 \%)$ & & & & \\
\hline
\end{tabular}

$S N P s$ single nucleotide polymorphisms, $N C R$ nonclopidogrel resistance, $C R$ clopidogrel resistance

Carriers were defined as patients with at least one CYP2C19 loss-of-function allele $(* 2$ or $* 3):{ }^{*} 1 / * 2, * 1 / * 3, * 2 / * 2, * 2 / * 3, * 3 / * 3$. Noncarrier was defined as patients without any CYP2C19 loss-of-function allele: wt/wt $\left({ }^{*} 1 /{ }^{*} 1\right.$ )

${ }^{*} P<0.05$

Bold values in tables are used to stress statistics with significant differences

\begin{tabular}{|c|c|c|c|c|c|c|c|c|}
\hline CYP2C19 & rs4244285/ rs4986893 & Carriers & $43(39.8 \%)$ & $65(60.2 \%)$ & 0.151 & 0.698 & 1.125 & $(0.621-2.035)$ \\
\hline \multirow{2}{*}{ NR112 } & rs13059232 & $\mathrm{CC}$ & 37 (55.2\%) & $30(44.8 \%)$ & 12.351 & $<0.001 * * *$ & 2.981 & $(1.606-5.533)$ \\
\hline & & $\mathrm{TT}+\mathrm{TC}$ & $36(29.3 \%)$ & 87 (70.7\%) & & & & \\
\hline
\end{tabular}

than those in the NCR group (Table 2, OR $=1.894,95 \% \mathrm{Cl}$ : $1.030-3.485, P=0.039$ ).

In phase 2, the validation cohort (clopidogrel cohort 2) comprised 140 patients from Guangdong General Hospital who consistently took clopidogrel; $32.9 \%(n=46)$ of these patients were considered to have poor outcomes. Clinical characteristics and clinical outcomes of the study population are shown in Table 1.

In phase 3, a cohort of 302 patients (aspirin cohort) who received a daily aspirin maintenance dose monotherapy during the 1-year follow-up was enrolled to compare the effects of selected SNPs on different antiplatelet agents; $30.8 \%(n=93)$ of these patients were considered to have poor outcomes. The clinical characteristics and clinical outcomes of the study population are shown in Table 1. There was no significant difference in age, gender, diabetes, smoking, or biochemical indexes between the clopidogrel and aspirin cohorts.

Phase 1: Association between CYP2C19*2/*3 and NR1/2 (rs13059232) gene polymorphisms and clopidogrel resistance Clopidogrel response was valued by the change in ADP-induced platelet aggregation before and after 5-7 days of treatment. Among 192 patients, there were three whose CYP2C19*2/*3 genotyping failed and two whose NR1/2 genotyping failed. In total, CYP2C19 *2/*3 genotyping of 189 patients and NR1/2 genotyping of 190 patients were successful. The allele frequencies of all SNPs were in Hardy-Weinberg equilibrium $(P>0.05)$. Supplementary Table S1 shows the frequency of alleles in our samples and in the PubMed database. Table 3 shows the association between the CYP2C19 and NR1/2 gene polymorphisms and CR. CYP2C19*2/*3 allele carriers had a higher risk of CR compared to noncarriers (carriers vs. noncarriers, OR $2.108,95 \% \mathrm{CI}$ 1.069-3.808, $P=0.029$ ). Regarding NR112 rs13059232, we first discuss the inheritance mode of this SNP. Supplementary Table S2 shows the comparisons of the genotype effects of NR1/2 rs13059232 with different modes of inheritance, confirming dominant inheritance. The CR in NR1/2 rs13059232 subjects with CC alleles was significantly greater than NR1/2 rs13059232 subjects with $\Pi$ and CT alleles (CC vs. $\Pi+T C$, OR 2.211, 95\% Cl $1.183-4.132, P=0.012)$. The logistic regression analysis showed that $C Y P 2 C 19^{*} 2 / * 3$ and the CC genotype of NR1/2 rs 13059232 were still two independent risk factors for the occurrence of $\mathrm{CR}$ after combining the CYP2C19 and NR1/2 genotyping results $(P=0.041, \operatorname{Exp}(B)=2.006 ; P=0.010, \operatorname{Exp}(B)=2.359$, respectively).

Phase 1: Association between NR1/2 (rs13059232) and clinical outcomes in clopidogrel cohort 1

To search for potential prognostic biomarkers of IS, we conducted an association analysis between the selected SNPs and clinical outcomes. Table 4 shows the correlation of selected SNPs with clinical outcomes. NR1/2 rs13059232 subjects with CC alleles had significantly worse clinical outcomes than patients with $\Pi$ and $C T$ alleles (Fig. 2a, CC vs. TT+TC, OR 2.981, 95\% Cl 1.606-5.533, $P<$ 0.001). However, none of the CYP2C19 SNPs correlated with clinical outcomes $(P>0.05)$. The logistic regression analysis showed that 
a

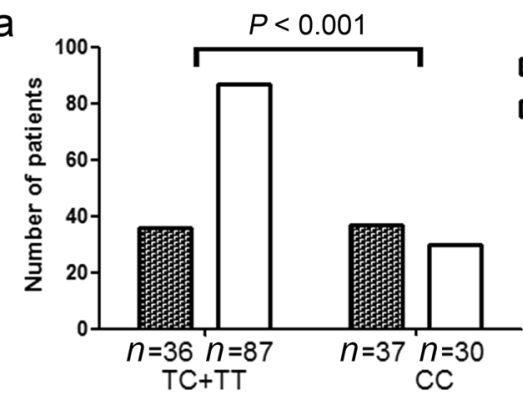

NR1I2 (rs13059232) \&clinical outcome in clopidogrel clohort 1

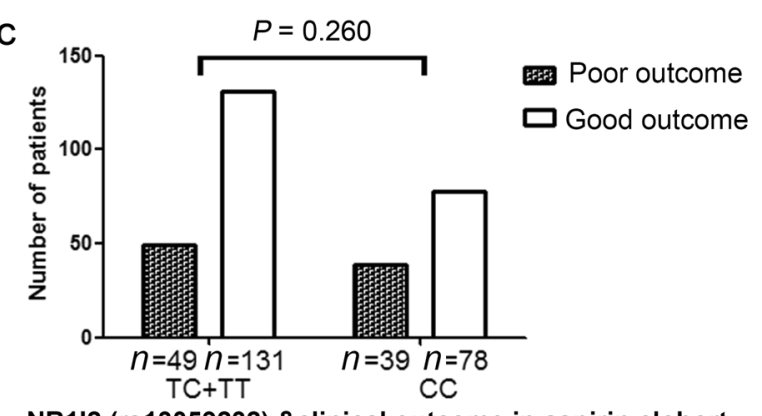

b

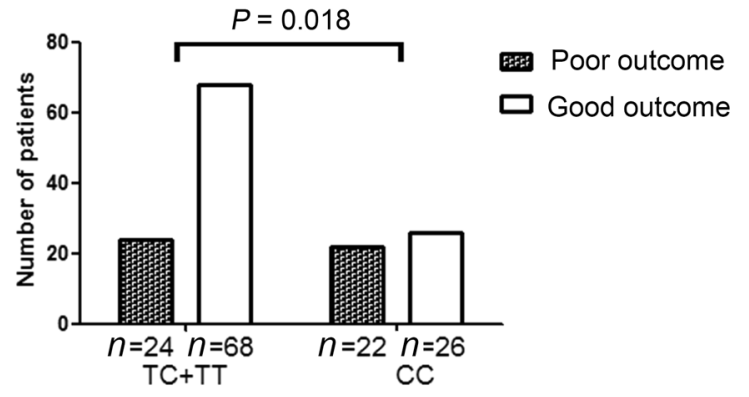

NR112 (rs13059232) \& clinical outcome in clopidogrel cohort 2

NR112 (rs13059232) \&clinical outcome in aspirin clohort

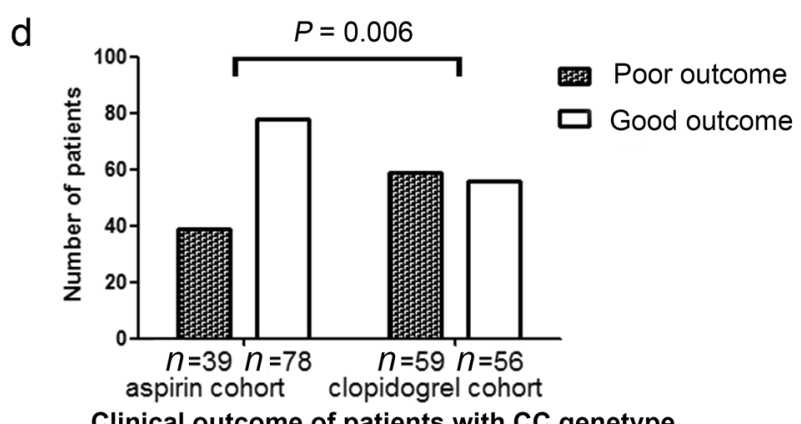

Fig. 2 Influences of SNP NR1/2 rs13059232 on the clinical outcomes of different antiplatelet drugs. a Association of NR1/2 rs13059232 with prognosis in a group of 192 patients from the Guangdong Provincial Hospital of Traditional Chinese Medicine $(P<0.001)$. b Association of NR1/2 rs13059232 with prognosis in a group of 140 patients from Guangdong General Hospital $(P=0.018)$. c Association of NR1/2 rs13059232 with prognosis in an aspirin group of 302 patients $(P=0.260)$. d Association of antiplatelet agents with prognosis in 193 patients with CC genotype $(P=0.006)$

the CC genotype of PXR rs13059232 was still an independent risk factor for the occurrence of poor clinical outcomes after combining the CYP2C19 and NR112 genotyping results $(P=$ $0.001, \operatorname{Exp}(B)=2.866)$.

Phase 2: Validation of results from phase 1

To further confirm the correlation between NR1/2(rs13059232) and clinical outcomes of IS patients who consistently took clopidogrel, we determined the NR112 genotypes (rs13059232) of an independent cohort of 140 patients to validate the results obtained in the last step. As shown in Fig. 2b, patients with the CC genotype had significantly worse clinical outcomes than patients with the CT/TT genotype (CC vs. TT+TC, OR 2.397, 95\% Cl 1.151-4.995, $P=0.018$ ). Thus, the results were validated in this independent cohort.

Phase 2: NR1/2 (rs13059232) and MACCEs

We also investigated the correlation between NR1/2 rs13059232 and MACCEs. During the 1-year follow-up, 80 (24.1\%) MACCEs occurred. Fig. 3 shows the results of a Kaplan-Meier analysis; patients with the CC genotype of NR1/2 rs13059232 had a significantly worse prognosis (CC vs. $C T+T, P=0.003$ ). Cox regression analysis suggested that the CC genotype of PXR rs13059232 and hypertension are independent risk factors for the occurrence of MACCEs $(P=0.003, \mathrm{HR}=1.922 ; P=0.018, \mathrm{HR}=$ 1.853 , respectively).

Phase 3: NR1/2 (rs13059232) and antiplatelet agent selection To determine whether NR1/2 (rs13059232) also affects the therapeutic effect of aspirin, we determined the effect of NR1/2 (rs13059232) on the clinical outcomes of IS patients who used aspirin as their secondary prevention antiplatelet drug. There was no significant correlation between the distribution of NR1/2 (rs13059232) and the clinical outcomes of patients taking aspirin (Fig. 2c, $P=0.260$ ). In addition, patients with the CC

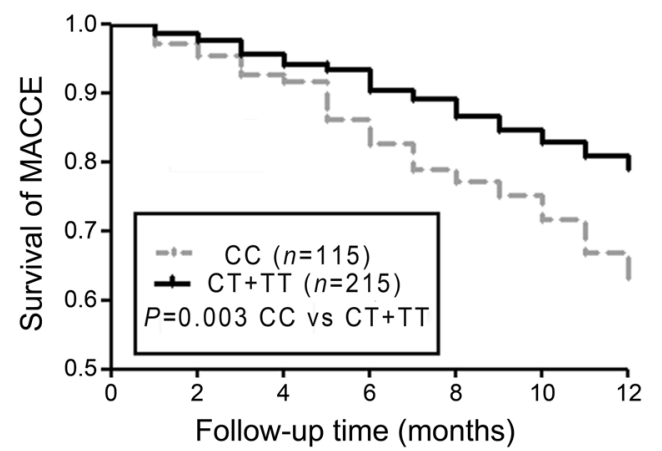

Fig. 3 Kaplan-Meier cumulative event rates of the MACCE (death, myocardial infarction, and additional. strokes) based on the different genotypes of SNP NR112 rs13059232 (CC vs. CT+TT, $P=0.003$ )

genotype who regularly took aspirin had significantly more favorable clinical outcomes than patients who regularly took clopidogrel (Fig. 2d, OR=2.107, $P=0.006$ ), which indicates that aspirin would be a rational choice for NR1/2 rs13059232 subjects with CC alleles.

\section{DISCUSSION}

In our study, by testing the MPAR of 192 patients with IS who took clopidogrel continuously for approximately 5-7 days, we found that NR1/2 rs13059232, CYP2C19*2/*3 and hypertension are independent risk factors for CR. The novel finding of this study is that during the 1-year follow-up period, NRII2 rs13059232 was identified as an independent risk factor associated with clinical outcomes in patients with acute IS who continued to take clopidogrel. Subjects with the CC genotype of NR1/2 rs13059232 had a higher rate of poor outcomes than subjects who were $T$ 
allele carriers (51.3\% vs. $28.0 \%$ ). Further comparisons were also made between IS patients who continuously took clopidogrel and those who took aspirin; this SNP influenced only the cohort of patients who continuously took clopidogrel. We also found that patients with the CC genotype who regularly took aspirin had significantly better clinical outcomes than those who continued to take clopidogrel during the 1-year follow-up $(\mathrm{OR}=2.107, P=$ $0.006)$. These new findings may provide guidance for selecting clopidogrel or aspirin as an antiplatelet treatment.

It is generally believed that CYP2C19 is the most significant factor contributing to the interindividual differences in clopidogrel responses [22]. Mutants of this gene, including CYP2C19*2 and CYP2C19*3, can decrease metabolic activity [23]. As a result, the transformation of clopidogrel to its active metabolite could be obstructed in patients with these mutants. Although the effect of CYP2C19*2/*3 has been confirmed in large-scale clinical studies [24], its association with clinical outcomes is in dispute. Some studies have indicated that CYP2C19 mutants cause poor clinical outcomes in patients, but this correlation was not found in some other studies [11, 25]. Other studies suggest that CYP2C19 allele carriers who consistently use clopidogrel in only the early stage have altered anticlopidogrel platelet reactivity and clinical outcomes $[26,27]$. In our current research, a significant correlation between CYP2C19*2/*3 and CR was found (CYP2C19*2/*3 alleles carriers vs. noncarriers, $\mathrm{OR} 2.108,95 \% \mathrm{Cl} 1.069-3.808, P=0.029)$, but we did not find a correlation between these mutations and long-term clinical outcomes.

NR1/2 (rs13059232) is an SNP located in intron 1 of NR1I2 [18]. In our previous studies, NR1/2 (rs13059232) was found to affect the occurrence of CR in IS patients treated with clopidogrel [28]. Studies have shown that the expression levels of NR1I2 could be affected by gene polymorphisms of NR112, which could further influence the activities of downstream metabolic enzymes and transporters $[20,21]$. For example, it was reported that $N R 1 / 2$ (rs13059232) influences the phenotypes of CYP3A4 [29], an important enzyme that metabolizes clopidogrel. Research has also found that the activation of PXR increases the xenobiotic metabolism of clopidogrel and decreases ADP-induced platelet aggregation [30]. Therefore, SNPs of NR1I2 may change drug efficacy by affecting related metabolic enzymes and transporters. A study from our research group showed that this SNP was correlated to variations in 4-OH-CPA plasma concentrations, which may be attributed to its regulation of CYP3A4 activity [31]. As a result, the activity of CYP3A4 may be downregulated in patients with the CC genotype of NR1/2 (rs13059232), and the transformation of clopidogrel to its active metabolite would be hindered, which could account for the higher rate of $C R$ and worse clinical outcomes compared with those of $\mathrm{T}$ allele carriers. Further studies are needed to investigate the influence of NR1I2 SNPs on the pharmacokinetic process of clopidogrel.

Apart from the effect of NR1/2 (rs13059232) on the pharmacokinetics of clopidogrel, the influence of this SNP on clinical outcomes may also be related to the extensive moderating effect of NR112 on other genes, such as antioxidant and antiatherosclerotic genes $[30,32]$. For example, research has shown that the upregulation of PXR protects vascular cells against oxidative damage by regulating the glutathione S-transferase (GST) family of phase II drug-metabolizing enzymes [30]. Moreover, a previous report showed that because the nuclear receptor PXR regulates lipid metabolism, the activation of PXR by PCN could decrease plasma LDL-cholesterol levels and prevent atherosclerosis [32].

By comparing the influence of NR1/2 (rs13059232) on patients taking antiplatelet drugs from different cohorts, our study provides a potential prognostic biomarker for determining the best medication for IS patients. Clopidogrel and aspirin are the most common antiplatelet drugs for the treatment and prevention of IS [2]. However, there is no consensus on the advantages and disadvantages of these two agents, and the clinical selection of antiplatelet agents for stroke treatment lacks evidence [33, 34]. In our research, the influence of NR1/2 (rs13059232) on clinical outcomes was found in the clopidogrel cohort but not in the aspirin cohort. This result may be because PXR regulates a variety of metabolic enzymes and transporters related to clopidogrel metabolism. In addition, in this study, patients with the CC genotype of rs13059232 who regularly took aspirin had significantly better clinical outcomes. Therefore, our results provide a novel genetic marker for determining the best individualized antiplatelet medication for patients with acute IS.

There are several limitations to our current study. (1) To further illustrate the influence of NR1/2 rs13059232 on patients' responses to clopidogrel, measurement of the pharmacokinetic process of clopidogrel and its active metabolite should be performed. (2) To improve the reliability of using NR1/2 rs13059232 as a prognostic biomarker for the selection of antiplatelet drugs for treating IS patients, a stricter prospective controlled trial is needed. (3) In addition to our three selected SNPs, many other genetic factors may influence the efficacy of clopidogrel. Therefore, a larger sample size and investigation of the influence of other genetic factors are needed.

In summary, this study showed a significant correlation between NR1/2 (rs13059232) and long-term clinical outcomes in IS patients who consistently took clopidogrel in two independent cohorts. Furthermore, the effect of this SNP on clinical outcomes was found to be affected by different antiplatelet drugs, which could be of great value in selecting individualized antiplatelet medications.

\section{ACKNOWLEDGEMENTS}

This work was supported by the National Major Projects for Science and Technology Development from the Science and Technology Ministry of China (Grant No. 2012ZX09506001-004), Guangdong Provincial Key Laboratory of Construction Foundation (No. 2017B030314030), the Guangzhou Science and Technology Project (No. 201604020003), the Guangdong Hospital of Chinese Medicine Project (YN2015B2025), and the Fundamental Research Funds for the Central Universities (No. 16ykpy14) and Science and Technology Planning Project of Guangdong Province (2017A020215147).

\section{AUTHOR CONTRIBUTIONS}

$\mathrm{Y}-\mathrm{bC}$ contributed to the study design, research performance, data analysis and manuscript writing; $\mathrm{J}, \mathrm{S}-\mathrm{IZ}$, and $\mathrm{Y}-\mathrm{fC}$ as the co-corresponding authors were involved in the study design, data collection, and final approval of the manuscript version to be published; Z-yZ and G-mL contributed to sample collection, acquisition of data, and experiments; $\mathrm{C}-\mathrm{xX}$ and $\mathrm{W}-\mathrm{bY}$ participated in experiment process, data analysis, and revision of the draft; $\mathrm{MH}$ contributed to the study design and data analysis.

\section{ADDITIONAL INFORMATION}

The online version of this article (https://doi.org/10.1038/s41401-018-0178-4) contains supplementary material, which is available to authorized users.

Competing interests: The authors declare no competing interests.

\section{REFERENCES}

1. Sacco RL, Diener HC, Yusuf S, Cotton D, Ounpuu S, Lawton WA, et al. Aspirin and extended-release dipyridamole versus clopidogrel for recurrent stroke. $\mathrm{N}$ Engl J Med. 2008;359:1238-51.

2. Kernan WN, Ovbiagele B, Black HR, Bravata DM, Chimowitz MI, Ezekowitz MD, et al. Guidelines for the prevention of stroke in patients with stroke and transient ischemic attack: a guideline for healthcare professionals from the American Heart Association/American. Stroke Assoc Stroke. 2014:45:2160-236.

3. Guthikonda S, Lev El, Kleiman NS. Resistance to antiplatelet therapy. Curr Cardiol Rep. 2005;7:242-8.

4. Fontana P, Dupont A, Gandrille S, Bachelot-Loza C, Reny JL, Aiach M, et al. Adenosine diphosphate-induced platelet aggregation is associated with P2Y12 gene sequence variations in healthy subjects. Circulation. 2003;108: 989-95. 
5. Lau WC, Waskell LA, Watkins PB, Neer CJ, Horowitz K, Hopp AS, et al. Atorvastatin reduces the ability of clopidogrel to inhibit platelet aggregation: a new drug-drug interaction. Circulation. 2003;107:32-7.

6. Lau WC, Gurbel PA, Watkins PB, Neer CJ, Hopp AS, Carville DG, et al. Contribution of hepatic cytochrome P450 3A4 metabolic activity to the phenomenon of clopidogrel resistance. Circulation. 2004;109:166-71.

7. Savi P, Combalbert J, Gaich C, Rouchon MC, Maffrand JP, Berger Y, et al. The antiaggregating activity of clopidogrel is due to a metabolic activation by the hepatic cytochrome P450-1A. Thromb Haemost. 1994;72:313-7.

8. Hollopeter G, Jantzen HM, Vincent D, Li G, England L, Ramakrishnan V, et al. Identification of the platelet ADP receptor targeted by antithrombotic drugs. Nature. 2001;409:202-7.

9. Savi P, Herbert JM, Pflieger AM, Dol F, Delebassee D, Combalbert J, et al. Importance of hepatic metabolism in the antiaggregating activity of the thienopyridine clopidogrel. Biochem Pharmacol. 1992;44:527-32.

10. Pan Y, Chen W, Xu Y, Yi X, Han Y, Yang Q, et al. Genetic polymorphisms and clopidogrel efficacy for acute ischemic stroke or transient ischemic attack: a systematic review and meta-analysis. Circulation. 2017;135:21-33.

11. Jia DM, Chen ZB, Zhang MJ, Yang WJ, Jin JL, Xia YQ, et al. CYP2C19 polymorphisms and antiplatelet effects of clopidogrel in acute ischemic stroke in China. Stroke. 2013;44:1717-9.

12. Price MJ, Murray SS, Angiolillo DJ, Lillie E, Smith EN, Tisch RL, et al. Influence of genetic polymorphisms on the effect of high- and standard-dose clopidogrel after percutaneous coronary intervention: the GIFT (Genotype Information and Functional Testing) study. J Am Coll Cardiol. 2012;59:1928-37.

13. Carlquist JF, Knight S, Horne BD, Huntinghouse JA, Rollo JS, Muhlestein JB, et al. Cardiovascular risk among patients on clopidogrel anti-platelet therapy after placement of drug-eluting stents is modified by genetic variants in both the CYP2C19 and ABCB1 genes. Thromb Haemost. 2013;109:744-54.

14. Gladding $\mathrm{P}$, White $\mathrm{H}$, Voss J, Ormiston J, Stewart J, Ruygrok $\mathrm{P}$, et al. Pharmacogenetic testing for clopidogrel using the rapid INFINITI analyzer: a doseescalation study. JACC Cardiovasc Interv. 2009;2:1095-101.

15. Su J, Xu J, Li X, Zhang H, Hu J, Fang R, et al. ABCB1 C3435T polymorphism and response to clopidogrel treatment in coronary artery disease (CAD) patients: a meta-analysis. PLoS ONE. 2012;7:e46366.

16. Tarkiainen EK, Holmberg MT, Tornio A, Neuvonen M, Neuvonen PJ, Backman JT, et al. Carboxylesterase 1 c.428G $>$ A single nucleotide variation increases the antiplatelet effects of clopidogrel by reducing its hydrolysis in humans. Clin Pharmacol Ther. 2015;97:650-8.

17. Shuldiner AR, O'Connell JR, Bliden KP, Gandhi A, Ryan K, Horenstein RB, et al. Association of cytochrome P450 2C19 genotype with the antiplatelet effect and clinical efficacy of clopidogrel therapy. JAMA. 2009;302:849-57.

18. Kliewer SA, Moore JT, Wade L, Staudinger JL, Watson MA, Jones SA, et al. An orphan nuclear receptor activated by pregnanes defines a novel steroid signaling pathway. Cell. 1998;92:73-82.

19. Brewer CT, Chen T. PXR variants: the impact on drug metabolism and therapeutic responses. Acta Pharm Sin B. 2016;6:441-49.

20. Chen Y, Tang Y, Guo C, Wang J, Boral D, Nie D. Nuclear receptors in the multidrug resistance through the regulation of drug-metabolizing enzymes and drug transporters. Biochem Pharmacol. 2012;83:1112-26.
21. Piedade R, Schaeffeler E, Winter S, Asimus S, Schwab M, Ashton M, et al. PXR variants and artemisinin use in Vietnamese subjects: frequency distribution and impact on the interindividual variability of CYP3A induction by artemisinin. Antimicrob Agents Chemother. 2012;56:2153-7.

22. Mega JL, Close SL, Wiviott SD, Shen L, Hockett RD, Brandt JT, et al. Cytochrome p-450 polymorphisms and response to clopidogrel. N Engl J Med. 2009; 360:354-62.

23. Kazui M, Nishiya Y, Ishizuka T, Hagihara K, Farid NA, Okazaki O, et al. Identification of the human cytochrome P450 enzymes involved in the two oxidative steps in the bioactivation of clopidogrel to its pharmacologically active metabolite. Drug Metab Dispos: Biol Fate Chem. 2010;38:92-9.

24. Mega JL, Close SL, Wiviott SD, Shen L, Walker JR, Simon T, et al. Genetic variants in $\mathrm{ABCB} 1$ and $\mathrm{CYP} 2 \mathrm{C} 19$ and cardiovascular outcomes after treatment with clopidogrel and prasugrel in the TRITON-TIMI 38 trial: a pharmacogenetic analysis. Lancet. 2010;376:1312-9.

25. Han Y, Lv HH, Liu X, Dong Q, Yang XL, Li SX, et al. Influence of genetic polymorphisms on clopidogrel response and clinical outcomes in patients with acute ischemic stroke CYP2C19 genotype on clopidogrel response. CNS Neurosci Ther. 2015;21:692-7.

26. Wallentin L, James S, Storey RF, Armstrong M, Barratt BJ, Horrow J, et al. Effect of CYP2C19 and $A B C B 1$ single nucleotide polymorphisms on outcomes of treatment with ticagrelor versus clopidogrel for acute coronary syndromes: a genetic substudy of the PLATO trial. Lancet. 2010;376:1320-8.

27. Campo G, Parrinello G, Ferraresi P, Lunghi B, Tebaldi M, Miccoli M, et al. Prospective evaluation of on-clopidogrel platelet reactivity over time in patients treated with percutaneous coronary intervention relationship with gene polymorphisms and clinical outcome. J Am Coll Cardiol. 2011;57:2474-83.

28. Liu R, Zhou ZY, Chen YB, Li JL, Yu WB, Chen XM, et al. Associations of CYP3A4, NR112, CYP2C19 and P2RY12 polymorphisms with clopidogrel resistance in Chinese patients with ischemic stroke. Acta Pharmacol Sin. 2016;37:882-8.

29. Lamba J, Lamba V, Strom S, Venkataramanan R, Schuetz E. Novel single nucleotide polymorphisms in the promoter and intron 1 of human pregnane $X$ receptor/NR1/2 and their association with CYP3A4 expression. Drug Metab Dispos: 2008;36:169-81.

30. Swales KE, Moore R, Truss NJ, Tucker A, Warner TD, Negishi M, et al. Pregnane X receptor regulates drug metabolism and transport in the vasculature and protects from oxidative stress. Cardiovasc Res. 2012;93:674-81.

31. Shu W, Guan S, Yang X, Liang L, Li J, Chen Z, et al. Genetic markers in CYP2C19 and CYP2B6 for prediction of cyclophosphamide's 4-hydroxylation, efficacy and side effects in Chinese patients with systemic lupus erythematosus. $\mathrm{Br} \mathrm{J}$ Clin Pharmacol. 2016;81:327-40.

32. Hoekstra M, Lammers B, Out R, Li Z, Van Eck M, Van Berkel TJ. Activation of the nuclear receptor PXR decreases plasma LDL-cholesterol levels and induces hepatic steatosis in LDL receptor knockout mice. Mol Pharm. 2009;6:182-9.

33. SPSS Group, Benavente OR, Coffey CS, Conwit R, Hart RG, McClure LA, et al. Blood-pressure targets in patients with recent lacunar stroke: the SPS3 randomised trial. Lancet. 2013;382:507-15.

34. Committee CS. A randomised, blinded, trial of clopidogrel versus aspirin in patients at risk of ischaemic events (CAPRIE). CAPRIE Steering Committee. Lancet. 1996;348:1329-39. 